\title{
Cell counting in the human brain: traditional and electronic methods*
}

\author{
J. A. N. Corsellis \\ F.R.C.P., F.R.C.Path., F.R.C.Psych.
}

\author{
R. L. Alston \\ F.I.M.L.T.
}

\author{
A. K. H. MiLleR \\ A.M.I.E.E.
}

\begin{abstract}
Department of Neuropathology, Runwell Hospital, Wickford, Essex
\end{abstract}
\begin{abstract}
Summary
Whether or not a proportion of nerve cells disappears from the human brain during adult life remains a controversial question 80 years after it was first asked. In this paper the authors review briefly the reasons for this uncertainty and describe an attempt to find an answer in so far as the cerebellar Purkinje cells are concerned. A loss of about $2.5 \%$ per decade was identified. The problems raised by cell counting in the cerebral cortex are outlined and a new method is described which may prove suitable for neuronal counting in these regions using an image analysing system.
\end{abstract}

IN 1894, Hodge, an American physiologist collected a number of young bees and an equal number of old ones, cut off their heads and compared their brains. The young ones were those crawling out of the brood cells; the old ones had worn frayed wings, roughened hair and 'bore the signs of extreme age'.

The old brains were found to be smaller than the young ones and cell counts showed a drop of nearly two out of every three cells. Hodge concluded that 'life starts with a superabundance of cells which gives the animal all the energy it needs but as the work of life is done, one by one, cells are worn out, finally the number falls too low and the functions of life cease'. Hodge made the bee study after he had found the number of Purkinje cells in the cerebellum of a man of 92 to be around $25 \%$ less than that in a man at 47 . He considered, however, that this single observation needed to be put on a firmer footing and in order to do this he had turned to the more systematic study of the bees.

Hodge appears to have been the first person to count nerve cells and to develop the concept of neuronal loss occurring with age. Since that time, which is now 80 years ago, the idea has broadened until it is now widely accepted that the decline in human intellect and performance with advancing age is the result of a loss of nerve cells from the

* The work is supported by the Medical Research Council as part of a Programme Grant. brain. Indeed, Delisle Burns epitomized this view in 1955 when he calculated that 100,000 nerve cells die? every day in the life of an adult man. Such ano hypothesis has its attractions, particularly for the young. It looks moreover as if it could well be truev since the loss of nervous tissue and of cortical nerve cells is often striking in the brains of organically? demented old people. It is then only a small step to $\overrightarrow{-}$ argue that such a pathological state is merely thes extreme of what is liable to happen in varyingo degrees to the population in general.

When, however, the literature on neuronal loss $8 n_{\overrightarrow{0}}$ ageing is examined, remarkably little quantitatie evidence can be found on which to base this assertion and in 1970 Konigsmark and Murphy inte duced a more sceptical attitude when they concluded that although there is loss of brain weight, volumeo and function in the later decades of life there is noo good evidence that this is due to neuronal loss. They $\mathbb{\Omega}$ had made this claim partly because they had found $\overrightarrow{\vec{\sigma}}$ no reduction with age in the cells of the human 3 ventral cochlear nucleus and partly because in their? view all previous studies had been based on samples. that were too small or on material that was un-o satisfactory.

It is the technical difficulties inherent in studying in sufficient detail a large enough number of indivi=duals that have blocked progress in this fleld for soo long. It is not surprising, therefore, that Hanley (1974), in a recent critical review of past work on the theory of neuronal fallout in ageing, could trace only twenty contributions during the present century. Half of these, moreover, were concerned withn experimental animals, which ranged from the housefly to the rat. Only one was based on a study of the cerebral cortex in man (Brody, 1955) while two, $\mathrm{E}$ apart from that of Hodge, had investigated the human cerebellar cortex (Ellis, 1920; Delorenzi, 1931).

There is a good reason for this preference, albeit ${ }^{+}$ slight, for working on the cerebellum rather than on $\frac{T}{0}$ the cerebral cortex. The Purkinje cells are large and $\stackrel{\oplus}{\stackrel{\circ}{\circ}}$ distinctive, they have well defined nucleoli and, above 
all, they are seen in suitably orientated histological sections, to lie in single file along a continuous line the length of which can be measured without great difficulty. It then becomes easy to assume, as Hodge and Delorenzi did, that it is necessary merely to count the number of cells lying along a measured length in order to derive for each brain the mean number of Purkinje cells per unit length of the Purkinje cell 'mono-layer'.

It is true that this is reasonably simple and a reliable method for studying variations in a cell population in different areas of the same cerebellum. Indeed, its use made it possible recently to identify and to quantify the way in which repeated blows to the head may lead to the destruction of a considerably greater number of Purkinje cells on the under surface of the cerebellum than over the dorsum (Corsellis, Bruton and Freeman-Browne, 1973).

A more complicated approach, however, is needed if reliable comparisons are to be made between cell populations in different cerebella. The reason for this may be illustrated by visualizing the sheet of Purkinje cells as an inflated balloon which is evenly covered in small dots. If the balloon loses air its volume decreases, its surface area shrinks and the dots (or cells) come closer together. In other words since the cerebellum, like the rest of the brain, varies in size from person to person or tends to become smaller with age, any neuronal loss will be masked by the drawing together of the remaining cells. In any study, therefore, it is advisable first to record the fresh cerebellar volume in each case, and secondly to measure the degree of shrinkage that takes place during the processing and cutting of any block of tissue. Corrections can then be applied which will allow for comparisons to be made between the cell populations of different individuals.

A technique for handling material in this way, which employed the use both of photography and of an image analysing system, was therefore evolved and the cerebella of ninety normal humans were examined.

The results of this study, which are being published in detail elsewhere (Hall, Miller and Corsellis, 1975) have shown that although appreciable differences occur from person to person at any given age. there is a tendency for the number of Purkinje cells to drop by about $2.5 \%$ per decade or by a total of $25 \%$ calculated over the age range of one to 100 years. The mean number of cells at all ages was found to be slightly lower in females than in males, but since the 'packing density' was the same, this difference was merely a reflection of the tendency for the male cerebellum to be larger than the female.

There now seems, therefore, reasonably good evidence to support the view that at least one major category of neurone, the Purkinje cell, is prone to die off in smallish numbers in the years before its owner does so. The more crucial question however still remains. This is whether the same is true, as Brody $(1955,1970)$ has claimed, of certain parts of the cerebral hemispheres, and in particular the frontal and temporal cortex. The practical problem is to find a reliable way of getting an answer, since the multilayered patterning of neurones of different shapes and sizes in the cortex, intermixed with the different types of glia, make any quantitative measurements by counting cells down a microscope excessively tedious and time-consuming, particularly if a large enough number of specimens and an adequate run of cerebral cortex are to be assessed reliably. Indeed, there are so many technical snags that it is scarcely surprising that Brody still stands alone in his painstaking attempts to quantify by eye, variations with age in the neuronal populations of the cerebral cortex.

To many people it has long seemed that the only solution to this problem would lie in the development of some kind of automatic cell-counting apparatus. J. Z. Young realized this more than 25 years ago when he pioneered the flying spot scanner. In this system a spot of light produced on the face of a cathode ray tube was used to scan the specimen in a series of lines and the amount of transmitted light was measured. In principle the grey level characteristic of objects in the specimen could then be reconstructed and individual cells recognized and counted. Unfortunately, problems of resolution and the complexity of the electronics necessary to carry out even such apparently simple tasks as reassembling individual cells from their partial images on subsequent scan lines, prevented any useful practical machine from being constructed. Technological developments over the last few years, particularly in the field of solid state digital electronics, have eliminated many of these problems. It is now possible, for example, to make use of devices which contain more than a thousand transistors and which are still less than a twentieth of a cubic inch in size. During the past few years several electronic machines, which take advantage of such developments, have become available and one of these has been found to be particularly suitable for the kind of work envisaged. This is the Quantimet 720 Image Analysing Computer which is capable of making feature-specific, as opposed to field-specific, measurements. That is to say, the automatic classification of each object or particle in a field on the basis of its individual characteristics is possible, instead of the mere provision of a measure of the mean characteristic for all objects in the field.

Figure 1 illustrates part of the machine. On the extreme left is the light microscope coupled to a television camera which displays on the adjacent 


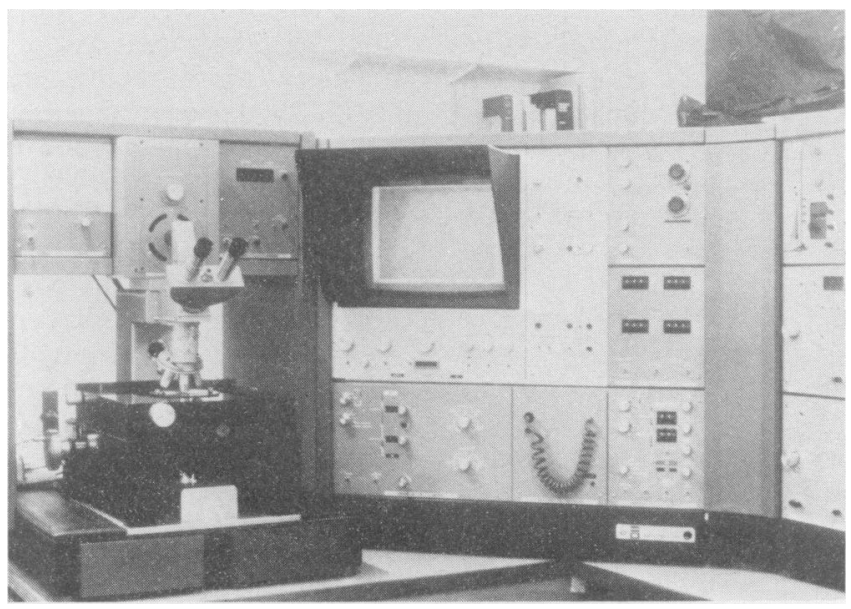

FIG. 1. Part of the Quantimet 720 Image Analysing Computer.

monitor screen the image of the field under the microscope. The stage is motor driven and can be programmed to move in minute, graduated steps in the $X$ and $Y$ directions, so that large areas may be scanned automatically in a series of adjacent fields.

Images, which appear on the monochrome monitor screen, can be detected on the basis of their grey levels - that is to say by how dark they appearby two controls on the detector module below the screen. Individual particles can therefore be picked out as long as they are darker or lighter than the general background. All subsequent operations will then only be carried out on particles so detected. It is possible to isolate simultaneously two different phases since there are two independently operating controls. Measurements which can be made on detected particles include area, perimeter, optical density and projection, and particles may be counted on the basis of any of these measurements or by a combination of them. Counting particles by combining a series of different parameters introduces an elementary form of pattern recognition. Thus if the machine is set to compute the ratio of area to the square of the perimeter it is possible to separate circles from triangles. Since, however, such precisely defined goemetric shapes are all too rare in biological tissue the ability to distinguish between them is of only limited application at present. This sort of problem emphasizes that such machines are still unable to solve many of the pattern recognition problems that are so trivial to the human eye and brain.

A second, more immediate, problem is the inability of the machine to separate automatically particles that touch or overlap so that a cluster of small objects cannot in general be distinguished from one large one. Because of this, it is essential to define $\vec{\circ}$ the precise technique for the preparation of specimens before presenting them to the machine. Particle응 overlap can be reduced to a minimum in human $\mathbb{\perp}$ brain tissue by making the histological sections th $\overrightarrow{0}$ enough. It is then necessary, however, to keep क्षे जे mind the danger that the staining density in to. thin a section will be reduced to an unacceptab?e level. Thicker sections may also introduce 'depth of field' problems for the microscope optics. Since the machine requires considerably higher contrast than $\frac{\mathscr{\Phi}}{\mathbb{Q}}$ the human eye for the correct detection of particles, $\cong$ counting is most accurate after careful compromise $\overrightarrow{\vec{O}}$ between section thickness and staining density; this 3 can only be achieved by experiment.

The technique used at present employs Carnoy-? fixed, $10 \mu \mathrm{m}$ paraffin-embedded sections stained with $\overline{0}$ quinolinic phthalocyanin (QPC) (Scott, 1972) using. an automatic slide staining machine. The method of $\frac{\sigma}{3}$ staining, which incorporates the critical electrolye concentration procedure described by Scott and $\frac{\rho}{2}$ Dorling (1965), allows detection of neuronal nucleoli at one grey level, by intense staining of RNA, $\frac{\text { ? }}{2}$ and detection of the surrounding nucleus and cyto- $\rightarrow$ plasm at another. (The highly specific, intense nucleic acid staining ability of QPC, with its particular N affinity for RNA and high resistance to extraction by processing solvents, points to an even wider 0 application in the field of biological image analysis $\omega$ than shown in this study.) This new dye, which is as yet commercially unavailable, emphasizes the strik- -0 ingly dark nucleoli together with a less intense, but even, staining of the neuronal cytoplasm against a ${ }^{+}$ remarkably homogeneous background. Since in most $\frac{0}{\circ}$ cell counting procedures only nucleolated neurones $\frac{\vec{D}}{\mathrm{D}}$ should be counted, the identification of nucleoli is $\underset{\mathbb{D}}{\stackrel{\mathrm{O}}{\mathrm{O}}}$ 


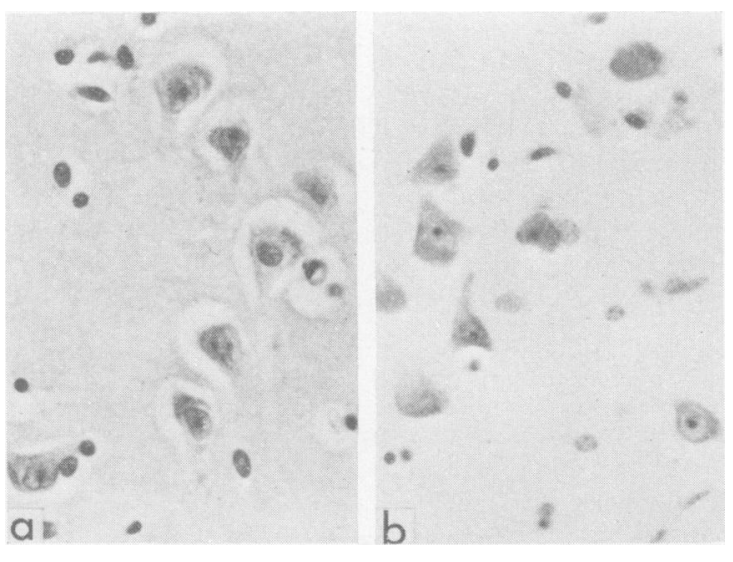

Fig. 2. Two fields of cerebral cortex; both $10 \mu$ sections embedded in paraffin. (a) Stained with cresyl violet; (b) stained with quinolinic phthalocyanin.

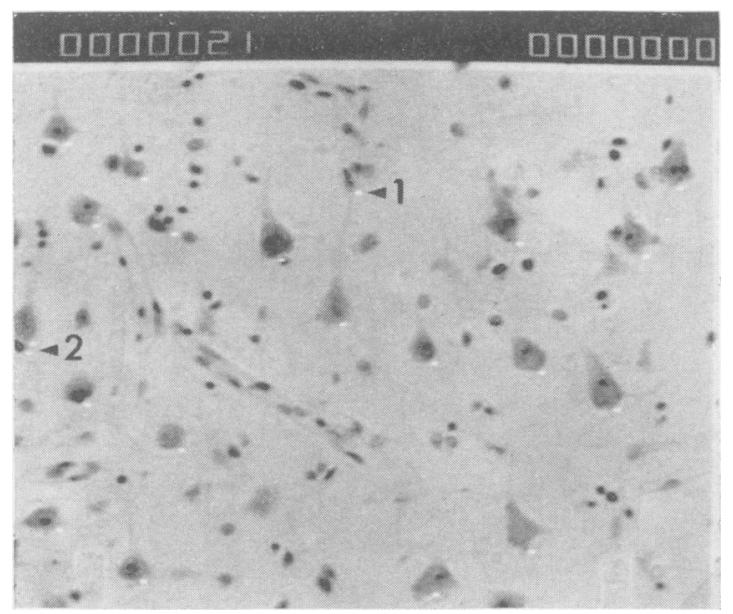

FIG. 3. An area of cortex as seen on the Quantimet screen, with count flags. Arrowhead 1 indicates a counting error; 2 points to an artefact.

crucially important. Figure 2 shows two fields, both from $10 \mu \mathrm{m}$ thick paraffin sections. The frame on the left (a) has been stained in the usual way with cresyl violet; that on the right (b) with QPC. In the latter, the background is clearer while the more even staining of the neuronal cytoplasm results in the more exact determination of its area. The nucleolus is also clearly demarcated from the nucleus. In Fig. 3 a QPC-stained section is shown on the monitor screen of the Quantimet. The number 0000021 in the top right hand corner is the machine's estimate of the number of neurones in the field irrespective of the presence of nucleoli. When the scanner has detected the lower boundary of a particle (i.e. of a cell) the stored number representing its measured area is

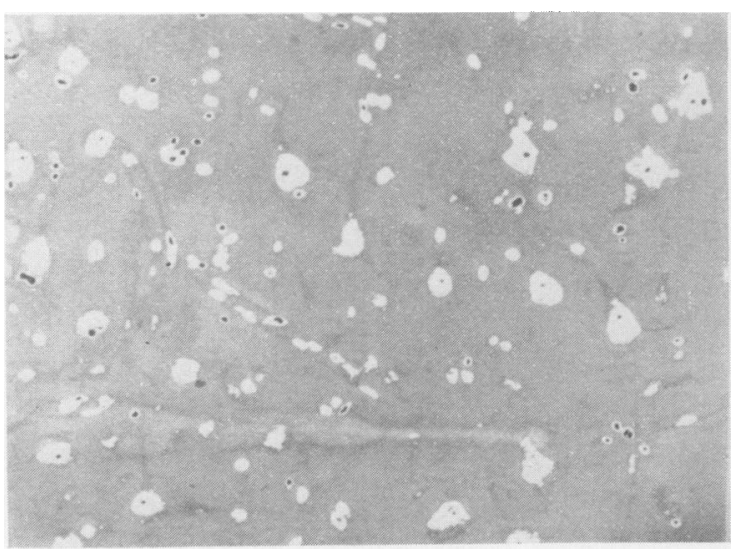

FIG. 4. A view of the Quantimet screen showing separate detection of cytoplasm and nucleoli.

first compared with the limits previously set up in the program. If the area of the particle is found by the machine to fall within these pre-set limits, a white counting flag appears at the bottom of the cell. These are visible in the illustration. Two points may be noted. Firstly, the arrowhead 1 points to a group of contiguous small cells which are being wronglo detected as a single large one. Secondly, there are twg cells on the extreme left of the picture (arrowhead with the suggestion of a double flag. This is a photographic artefact due to the length of exposure and electronic vacillation. The total count will not be affected.

The counting of nerve cells in this way, however, is only the first step. In order to obtain an estimate of the total number of neurones in a given volume of material, it is preferable to count only nucleolated cells since this greatly reduces the likelihood of counting the same cell in adjacent sections. This has been achieved by detecting nucleoli and cytoplasm separately, and only counting cells if the cytoplasmic area falls within specified limits and if there is a $\delta$ nucleolus contained within the cytoplasm. Figure 4 shows detection on this basis, the nucleoli appearing $ᄋ$ dark on the monitor screen. This method has another advantage in that where previously clusters of, for instance, oligodendroglial cells, which appear very dark in this stain, were counted as neurones; now, o since the area discrimination is performed only on $\mathbb{O}$ the lighter grey areas, these cells are rejected. Figure స్ల 5 shows the machine counting nucleolated neurones. O्व There are now only two errors, the first marked ' 1 ', where a cluster of glial cells has been counted, and the second marked ' 2 ' where it is counting a nonnucleolated nerve cell which is in contact with a dark 0 glial cell. Nerve cells without nucleoli, such as that marked ' 3 ', are not being counted. 


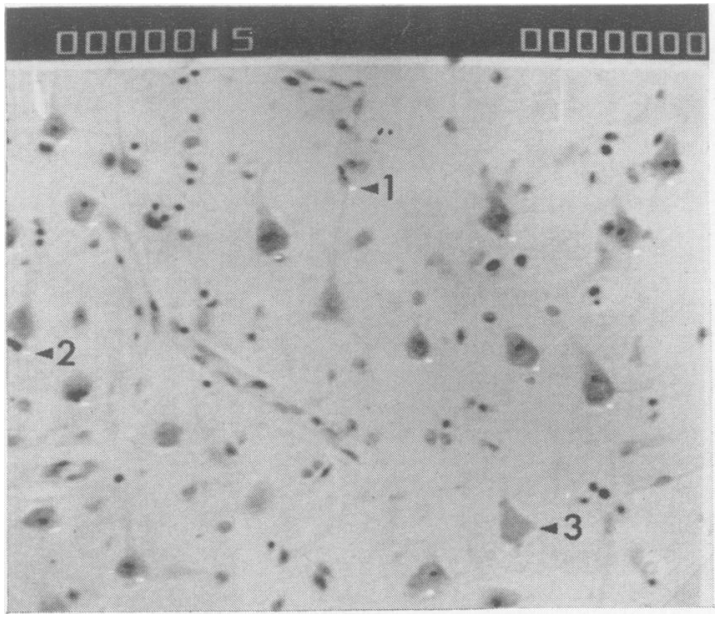

FIG. 5. A view of the screen with only nucleolated neurons being counted. Arrowhead 1 illustrates an eror; 2 points to the same artefact as in Fig. $3 ; 3$ is a nonnucleolated neurone and therefore without a flag.

For automatic operation all that is required is for the detection level to be set on the first field. Cells will then be counted at a rate of about one field per second $(90 \%$ of this time being taken moving the stage and allowing it to settle), and the results passed out to a programmable calculator, which stores them and organizes them in the way its programme dictates. At the end of a run, which could consist of several hundred fields, the results are printed out and, if required, a histogram of cell distribution plotted automatically. The area of the cells in each field is also available.

The main advantage of the machine in cell counting is its speed of operation. Although its accuracy may be less than that of the human observer this disadvantage is more than outweighed by the much greater volume of material which can be handled. This is particularly important in studies concerned primarily with comparative counts. Since it may be assumed that the errors will be similar in all the $\stackrel{\mathbb{Q}}{\varrho}$ material studied relatively small differences will still. be detected.

The work has now reached the stage at which the techniques have been established. The next step is to begin to identify the extent of any differences that $\frac{\overline{\bar{n}}}{\bar{n}}$

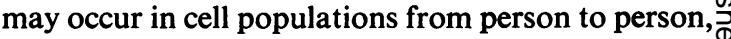
with adancing age, and in the presence of disease.

\section{Acknowledgments}

We are particularly grateful to Dr J. E. Scott for supplying us so generously with the dye, quinolinic phthalocyanin. His $\vec{\omega}$ help and advice at all times have been invaluable.

\section{References}

BRODY, H. (1955) Organization of the cerebral cortex. III. Acr study of ageing in the human cerebral cortex. Journal of Comparative Neurology, 102, 511.

BRoDY, H. (1970) Structural changes in the ageing nervous. system. Interdisciplinary Topics in Gerontology, 7, 9.

Corsellis, J.A.N., Bruton, C.J. \& Freeman-Browne, D.N (1973) The aftermath of boxing. Psychological Medicine, 3, 음 270.

Delorenzi, E. (1931) Costanza numerica delle cellule di $\overrightarrow{0}$ Purkınje del cervelletto dell'uomo in individui di varia età. Zeitschrift für Zellforschung und mikroskopische Anatomie, 14, 310.

ELLIS, R.S. (1920) Norms for some structural changes in the human cerebellum from birth to old age. Journal $8 f_{6}$ Comparative Neurology, 32, 1.

Hall, T.C., Miller, A.K.H. \& Corsellis, J.A.N. (19) Variations in the human Purkinje cell population accore ing to age and sex. Neuropathology and Applied Neurobiology, 1, 267.

HANLEY, T. (1974) 'Neuronal fall-out' in the ageing brain: a critical review of the quantitative data. Age and Ageing, 3, 133.

HoDGE, C.F. (1894) Changes in ganglion cells from birth to senile death. Observations on man and honey-bee. Journalo of Physiology, 17, 129.

Konigsmark, B.W. \& MurPhy, E.A. (1970) Neuronal populations in the human brain. Nature, London, 228, 1335.

ScOTT, J.E. (1972) Histochemistry of alcian blue. III. The molecular biological basis of staining by alcian blue $8 \mathrm{GX}$. and analogous phthalocyanins. Histochemie, 32, 212.

ScoTT, J.E. \& Dorling, J. (1965) Differential staining of? acid glycosaminoglycans (mucopolysaccharides) by alcian blue in salt solutions. Histochemie, 5, 221. 